\title{
On the origin of the $\mathrm{A}_{1 g}$ and $\mathrm{B}_{1 g}$ electronic Raman scattering peaks in the superconducting state of $\mathrm{YBa}_{2} \mathrm{Cu}_{3} \mathrm{O}_{7-\delta}$
}

\author{
H. Martinho, ${ }^{1}$ A. A. Martin, ${ }^{2}$ C. Rettori, ${ }^{1}$ C. T. Lin,${ }^{3}$ and C. Bernhard, ${ }^{3}$ \\ ${ }^{1}$ Instituto de Física "Gleb Wataghin", UNICAMP, 13083-970, Campinas, SP, Brazil \\ ${ }^{2}$ Instituto de Pesquisa e Desenvolvimento - UNIVAP, \\ 12 244-050, São José dos Campos, SP, Brazil and \\ ${ }^{3}$ Max-Planck-Institut für Festkörperforschung, \\ Heisenbergstrasse 1, D-70 569 Stuttgart, Germany.
}

\begin{abstract}
The electronic Raman scattering has been investigated in optimally oxygen doped $\mathrm{YBa}_{2} \mathrm{Cu}_{3} \mathrm{O}_{7-\delta}$ single crystals as well as in crystals with non-magnetic, $\mathrm{Zn}$, and magnetic, $\mathrm{Ni}$, impurities. We found that the intensity of the $\mathrm{A}_{1 g}$ peak is impurity independent and their energy to $T_{c}$ ratio is almost constant $\left(2 \Delta / k_{B} T_{c} \sim 5\right)$. Moreover, the signal at the $\mathrm{B}_{1 g}$ channel is completely smeared out when non-magnetic $\mathrm{Zn}$ impurities are present. These results are qualitatively interpreted in terms of the Zeyher and Greco's theory that relates the electronic Raman scattering in the $\mathrm{A}_{1 g}$ and $\mathrm{B}_{1 g}$ channels to $d$-CDW and superconducting order parameters fluctuations, respectively.

PACS numbers: 78.30.-j;74.25.-q;74.25.Gz;74.72.-h
\end{abstract}

Far a long time, the phenomenon of high- $T_{c}$ superconductivity in the cuprates has been considered as related to an unconventional pairing state. [1] It is widely accepted that the unconventional mechanism in these materials is closely related to their normal state properties, e.g., the non-Fermi-liquid behavior, 22 in spite of the lack of consensus about the correct description of this state. The presence of several kinds of fluctuations, such as spin, flux phases, stripes or charge density waves (CDW) is an additional complication $[3$ and many models considering one or more of these fluctuations have been proposed. However, there is a large amount of experimental data that these models cannot explain (see, e.g., sections 5.4 and 6.4 of Refs. 1] and 2], respectively). Among these data we mention the absence of a convincent explanation for the electronic Raman scattering (ERS) of many cuprates in the superconducting state.

The redistribution of the ERS in the superconducting state has been used to study the gap order parameter in many superconductors, both conventional or unconventional, such as $\mathrm{Nb}_{3} \mathrm{Sn},[4]$ cuprates, $[5]$ borocarbides $[\underline{6}$ and recently, $\mathrm{MgB}_{2}$. 7] In general, the ERS of the cuprates presents two characteristic peaks in the $\mathrm{B}_{1 g}$ and $\mathrm{A}_{1 g}+\mathrm{B}_{2 g}$ channels in the superconducting phase that disappear above $T_{c}$. In the $\mathrm{YBa}_{2} \mathrm{Cu}_{3} \mathrm{O}_{7-\delta}(\mathrm{Y} 123)$ system the ERS of optimally doped crystals has shown the $\mathrm{A}_{1 g}$ peak stronger than the $\mathrm{B}_{1 g}$ counterpart. Also, the maximum intensity of the $\mathrm{A}_{1 g}$ peak lies at a lower energy than that of the $\mathrm{B}_{1 g}$ one. [8] In the overdoped state, the $\mathrm{B}_{1 g}$ peak shifts down converging approximately to the same position of the $\mathrm{A}_{1 g}$ peak [8] while in the strongly underdoped regime neither $\mathrm{A}_{1 g}$ nor $\mathrm{B}_{1 g}$ peaks are observed.

The appearance of these peaks only below $T_{c}$, leads one to believe that they are related to the superconductivity, probably being pair-breaking peaks. Some publications [9] have already shown that, for a gap with nodes and $d_{x^{2}-y^{2}}$ order parameter at low energies, the ERS efficiency obeys the $\sim \omega^{3}$ and $\sim \omega$ power-laws in the
$\mathrm{B}_{1 g}$ and $\mathrm{A}_{1 g}+\mathrm{B}_{2 g}$ channels, respectively. Besides, these works have predicted that the $\mathrm{A}_{1 g}$ peak should be weaker than the $\mathrm{B}_{1 g}$ component with their maxima appearing at the same energy $2 \Delta$. In spite of the good agreement between the theory and the experimentally observed power laws, the relative position and intensity of the ERS peaks are in disagreement with the theoretically expected results for the pair-breaking ERS response. Hence, the origin of the $\mathrm{A}_{1 g}$ and $\mathrm{B}_{1 g}$ peaks remains unclear.

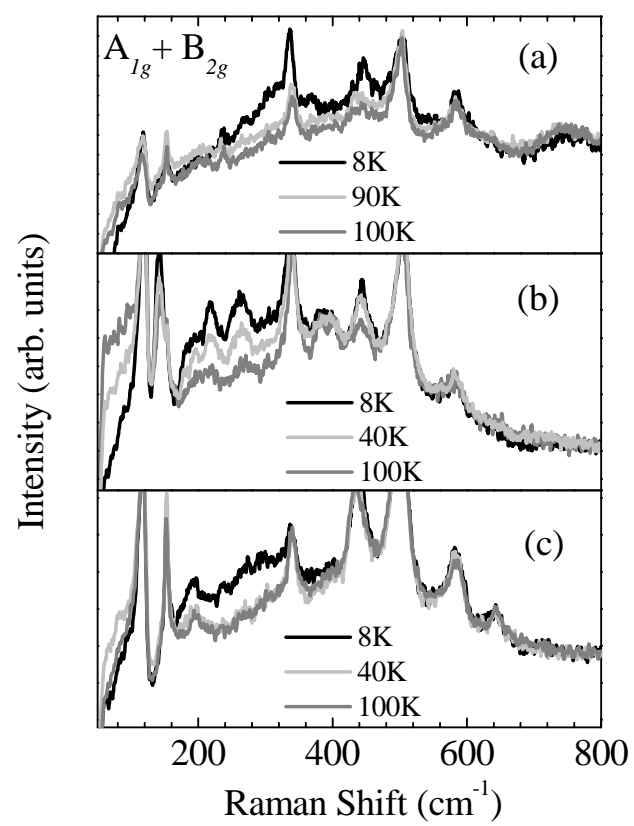

FIG. 1: Temperature dependence of the Raman spectra in the $\mathrm{A}_{1 g}+\mathrm{B}_{2 g}$ channel showing the redistribution of the ERS below $T_{c}$ for (a) Y123 with $\mathrm{T}_{c}=91 \mathrm{~K}$, (b) Y123:Ni with $\mathrm{T}_{c}=76 \mathrm{~K}$, and (c) Y123:Zn with $\mathrm{T}_{c}=72 \mathrm{~K}$ single crystals.

In this letter we make an attempt to clarify this ques- 
tion by means of ERS measurements in Y123 single crystals doped with a small amount, 5\%, of either, magnetic $\mathrm{Ni}^{2+}(\mathrm{Y} 123: \mathrm{Ni})$ or non-magnetic $\mathrm{Zn}^{2+}(\mathrm{Y} 123: \mathrm{Zn})$ impurities in the copper planes. It is known that substituting $\mathrm{Cu}$ by $\mathrm{Zn}$ or $\mathrm{Ni}$ in Y123 preserves the oxygen doping level and modify only slightly the crystal structure. Also, the non-magnetic $\mathrm{Zn}^{2+}$ impurities, contrary to the magnetic $\mathrm{Ni}^{2+}$ ones, restore significant spin fluctuations in the normal state. 10, 11] Since the presence of impurities in the $\mathrm{CuO}_{2}$ planes are known to induce a pair-breaking effect, 10, 11] the investigation of the change in the electronic properties by introducing magnetic and non-magnetic impurities can give an important insight into the open questions related to the physics of the cuprates.

Single crystals of Y123, Y123:Ni and Y123:Zn were prepared as previously described. 12] After a thermal treatment, the transition temperatures were measured by $d c$-magnetization and were found to be 91,76 and 72 $\mathrm{K}$ for the Y123, Y123:Ni and Y123:Zn samples, respectively. In terms of the oxygen level, all samples are in the optimally-doped state. The Raman measurements were carried out using a triple spectrometer equipped with a $\mathrm{LN}_{2}$ CCD detector. The $514.5 \mathrm{~nm}$ line of an $\mathrm{Ar}^{+}$ion laser was used as an excitation source. The laser power at the sample was kept below $8 \mathrm{~mW}$ on a spot diameter of about $50 \mu \mathrm{m}$. The samples were cooled in an exchange He gas variable temperature cryostat, and measured in a nearbackscattering configuration on the $a b-$ plane. For the tetragonal symmetry $D_{4 h}$, the choice of the $x^{\prime}, x^{\prime}$ geometry probes a combination of the $\mathrm{A}_{1 g}$ and $B_{2 g}$ symmetries, while choosing the $x^{\prime}, y^{\prime}$ geometry couples to excitations of $\mathrm{B}_{1 g}$ symmetry. $x^{\prime}\left(y^{\prime}\right)$ denote axes rotated by $45^{\circ}$ from the crystallographic $x(y)$ axes.

In Fig. 1 we present the Raman spectra in the $\mathrm{A}_{1 g}+\mathrm{B}_{2 g}$ channel at different temperatures for the Y123, Y123:Ni and Y123:Zn samples, corrected by the thermal BoseEinstein factor. In Fig. 1(a) the spectrum for Y123 at $100 \mathrm{~K}$ displays a flat background and just below $\mathrm{T}_{c} \sim 91$ $\mathrm{K}$ starts the rearrangement of the electronic background, resulting in a broad peak in the spectral range between 200 and $400 \mathrm{~cm}^{-1}$. The same behavior is also found in Fig.1 (b) for Y123:Ni. However, in this case the rearrangement of the ERS starts only below $50 \mathrm{~K}$, producing a broad peak located in the same spectral range as far the pure sample. For Y123:Zn, Fig.1(c), the gain of spectral weight in the superconducting state is also present in the $\mathrm{A}_{1 g}+\mathrm{B}_{2 g}$ channel, although it starts to appears at 40 $\mathrm{K}$, below $\mathrm{T}_{c} \sim 72 \mathrm{~K}$, but is located in the same spectral range as the other two samples.

In the $\mathrm{B}_{1 g}$ channel, Fig.2, the rearrangement of the ERS is also displayed for Y123 and Y123:Ni. For Y123, Fig.2(a), it starts below $70 \mathrm{~K}$ and it appears in the 450 to $650 \mathrm{~cm}^{-1}$ spectral range. For Y123:Ni, Fig.2(b), the broad peak first appears below $60 \mathrm{~K}$ and it is also located in the 450 to $650 \mathrm{~cm}^{-1}$ spectral range. Surprisingly, for Y123:Zn, Fig. 2(c), the rearrangement of the ERS is absent below $T_{c}$. The only observed effect of lowering

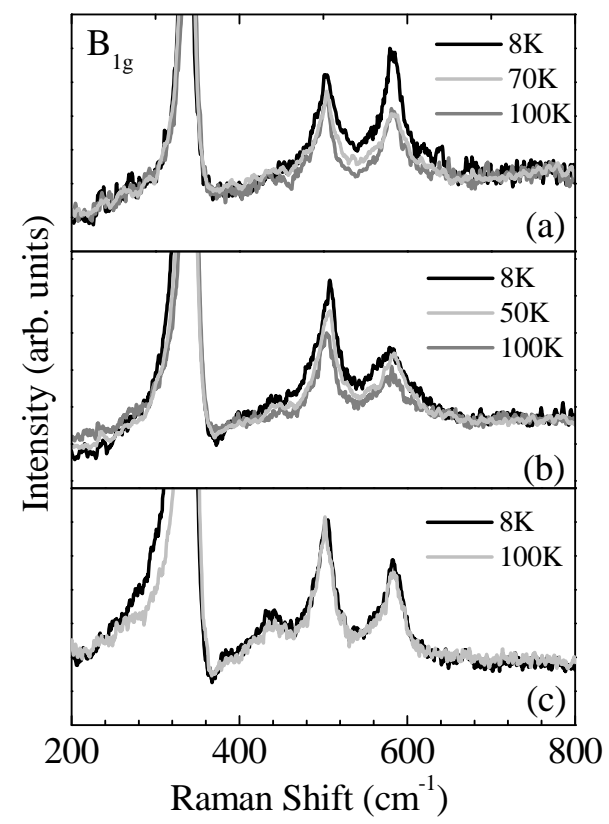

FIG. 2: Temperature dependence of the polarized Raman spectra in $B_{1 g}$ channel for (a) Y123, (b) Y123:Ni and (c) Y123:Zn.

the temperature is the broadening of the $\mathrm{B}_{1 g}$ phonon at $330 \mathrm{~cm}^{-1}$.

In order to determine the energy of the broad peaks which appear in the superconducting phase in the $\mathrm{A}_{1 g}+\mathrm{B}_{2 g}$ and $\mathrm{B}_{1 g}$ channels, the pure ERS response function has been obtained by subtracting the contribution of the phonons fitted to Lorentzian or Fano-profiles to the Bose-Einstein corrected raw data.

In Figure 3 we present the pure ERS response for the $\mathrm{A}_{1 g}+\mathrm{B}_{2 g}$ channel at 8 and $100 \mathrm{~K}$. At $8 \mathrm{~K}$ we found the $\mathrm{A}_{1 g}$ response peaks around 320,250 and $300 \mathrm{~cm}^{-1}$ for Y123 (3a), Y123:Ni (3b) and Y123:Zn (3c), respectively. We notice that the $\mathrm{A}_{1 g}$ energy to $T_{c}$ ratio $\hbar \omega_{A_{1 g}} / k_{B} T_{c}$ is $\sim 5.0$ for $\mathrm{Y} 123, \sim 4.7$ for $\mathrm{Y} 123: \mathrm{Ni}$, and $\sim 5.8$ for $\mathrm{Y} 123: \mathrm{Zn}$. As commented above, at $100 \mathrm{~K}$, the superconducting rearrangement of the ERS is absent and the Raman spectra present no pronounced peak.

Figure 4 shows the pure ERS for the $\mathrm{B}_{1 g}$ channel at 8 and $100 \mathrm{~K}$. The dramatic difference between the response of Y123:Zn compared with that of the other crystals is evident. The peak is absent in Y123:Zn but it appears centered around $480 \mathrm{~cm}^{-1}$ in Y123 and Y123:Ni. The ratio $\hbar \omega_{B_{1 g}} / k_{B} T_{c}$ is $\sim 7.7$ for Y123 and $\sim 9.2$ for Y123:Ni. Moreover, the ERS spectrum in Y123:Zn is almost the same at 8 and $100 \mathrm{~K}$ being also very similar to those in Y123 and Y123:Ni at $100 \mathrm{~K}$, except for a small difference in the absolute value of the intensities.

Observation of Fig. 3 and 4 indicates that the Zn substitution affects the $\mathrm{A}_{1 g}+\mathrm{B}_{2 g}$ and $\mathrm{B}_{1 g}$ channels in a quite different way. While in the $\mathrm{A}_{1 g}+\mathrm{B}_{2 g}$ channel the intensities of the peaks are almost unaffected by the impurities, 


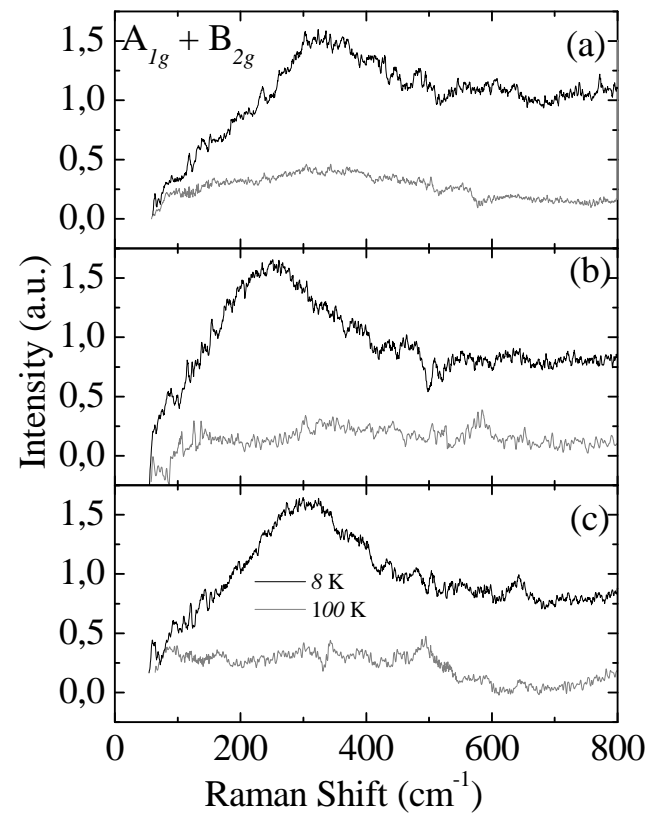

FIG. 3: Electronic Raman response at $8 \mathrm{~K}$ (black lines) and $100 \mathrm{~K}$ (gray lines) for (a)Y123, (b) Y123:Ni, and (c)Y123:Zn single crystals in $\mathrm{A}_{1 g}+\mathrm{B}_{2 g}$ channel.

in the $\mathrm{B}_{1 g}$ channel the peak is smeared out in the $\mathrm{Zn}$ doped sample. The comparison between the $\mathrm{B}_{1 g}$ channel signals at 8 and $100 \mathrm{~K}$ in Y123:Zn indicates that the ERS in the superconducting and normal states are nearly the same. This unusual effect of $\mathrm{Zn}$ substitution on the $\mathrm{B}_{1 g}$ Raman response is surprising and has not been predicted by any theoretical model nor been observed by other systematic experimental work. Another relevant result is the almost constant energy to $T_{c}$ ratio $\sim 5-6$ for the $\mathrm{A}_{1 g}$ peak for all samples. This value is in agreement with previous values obtained for the $\mathrm{A}_{1 g}$ peak 13 , 16] and for the superconducting gap measured by electron tunneling spectroscopy in Y123. 14

Recently, Venturini et al [15] and Zeyher and Greco [17] have discussed theoretically the ERS in the superconducting phase of high- $T_{c}$ cuprates.

The work of Venturini et al. 15 presents a model suggesting that the observed peak in the $\mathrm{A}_{1 g}+\mathrm{B}_{2 g}$ channel is produced by collective spin fluctuations, being a twomagnon Raman peak. In the same model, the $\mathrm{B}_{1 g}$ peak is related to pair breaking. They were able to fit the experimental Raman spectrum of Bi2212 to their model and obtained the correct relative position of the $\mathrm{A}_{1 g}$ and $\mathrm{B}_{1 g}$ peaks. 15] Moreover, Gallais et al. 16] have shown that the $\mathrm{A}_{1 g}$ peak tracks the magnetic resonance peak observed by inelastic neutron scattering [10] at $40 \mathrm{meV}$ in Ni-substituted $\mathrm{YBa}_{2} \mathrm{Cu}_{3} \mathrm{O}_{6.95}$. These authors have interpreted this fact as an evidence for the magnetic origin of the $\mathrm{A}_{1 g}$ peak.

However, our results cannot be interpreted in these terms. The main reason is the strong Zn-impurity de-

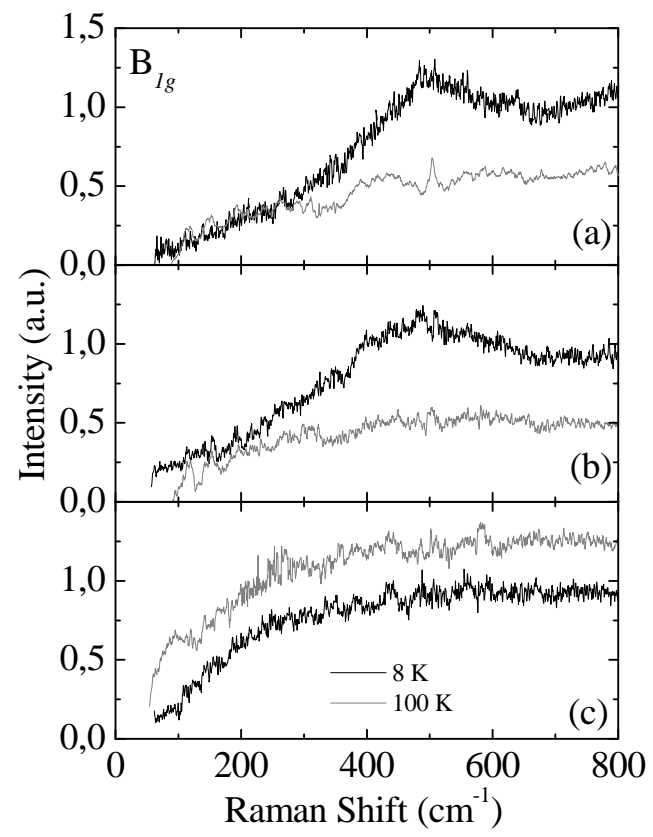

FIG. 4: Electronic Raman response at $8 \mathrm{~K}$ (black lines) and $100 \mathrm{~K}$ (gray lines) for (a) Y123, (b) Y123:Ni, and (c) Y123:Zn in the $\mathrm{B}_{1 g}$ channel.

pendency observed in the $\mathrm{B}_{1 g}$ spectra. In the Venturini's framework the $\mathrm{B}_{1 g}$ peak is related to pair-breaking process with their maximum at $2 \Delta$ energy. Thus, the complete smearing out of the $\mathrm{B}_{1 g}$ peak observed in our experiments (see Fig. $4 \mathrm{c}$ ) would imply that $2 \Delta \rightarrow 0$ for the Zn-substituted crystal. However, it is known that $T_{c}$ does not go to zero in this case and, discharging any anomalous behavior of the $2 \Delta / k_{B} T_{c}$ ratio, $2 \Delta \nrightarrow 0$. Futhermore, the $\hbar \omega_{A_{1 g}} / k_{B} T_{c}$ ratio for the $A_{1 g}$ peak presents better agreement to the gap energy measured by others groups (see, e.g., ref. 14]) than the $\mathrm{B}_{1 g}$ one, indicating that the $\mathrm{A}_{1 g}$ ERS peak is related to pair-breaking.

Another possible theoretical comparison could be made with the Zeyher and Greco's 17 theory. These authors used the superconducting model by Cappelluti and Zeyher [18] in order to understand the ERS of cuprates. The Cappelluti and Zeyher model proposed that the superconductivity in cuprates is originated by the competition between the superconducting and the $d$-CDW (also called orbital antiferromagnetism) order parameters. Zeyher and Greco [17] suggested that the $\mathrm{A}_{1 g}$ and $\mathrm{B}_{1 g}$ peaks are caused by amplitude fluctuations of the superconducting and $d$-CDW order parameters, respectively. The $d$-CDW phase corresponds to a flux phase where current flows around each $\mathrm{CuO}_{2}$ square alternatively clockwise and counterclockwise 19 giving rise to orbital antiferromagnetism where the only interaction present in the order parameter is the Heisenberg exchange coupling between the $\mathrm{Cu}^{2+}$ ions. 17.

As mentioned above, our experimental data indicates that the $\mathrm{A}_{1 g}$ peak is related to pair-breaking. In this 
sense, our experimental ERS results give support to the Zeyher and Greco's theory. Nevertheless, the impurity effect on the $d$-CDW $\mathrm{B}_{1 g}$ peak is not well theoretically understood at the moment. Cappelluti and Zeyher 21] have shown that the Zn impurities substitution does not have appreciable influence on the oxygen doping phase diagram of the cuprates. However, the effect on the $\mathrm{B}_{1 g}$ ERS signal could be more subtle and a more detailed theoretical calculation could be used.

Nonetheless, qualitatively we can elaborate about the impurity substitution effect on $\mathrm{B}_{1 g}$ signal as follows. It is known that the main interaction originating the $d$ CDW is the Heisenberg coupling between the $\mathrm{Cu}^{2+}$ spins. Thus, it is expected that the $\mathrm{Cu}^{2+}(S=1 / 2)$ substitution by $\mathrm{Zn}^{2+}(S=0)$ impurity, would break down the long range coherence of the orbital antiferromagnetism, explaining the disappearence of the $d$-CDW excitation in the $\mathrm{B}_{1 g}$ channel. In fact, this is consistent with the larger depletion of $T_{c}$ just produced by the presence of $\mathrm{Zn}^{2+}$ impurities. 10, 11] Besides, the $\mathrm{Ni}^{2+}(S=1)$ impurities could be less effective in breaking down the orbital antiferromagnetism notwithstanding having also effect in decreasing $T_{c}$. Moreover, as shown by Gupta and Gupta [20] the charge density redistribution due to the $\mathrm{Ni}^{2+}$ ions is localized while the $\mathrm{Zn}^{2+}$ perform an extended perturbation. On this basis, one would expect that the $\mathrm{Zn}^{2+}$ ions would be more effective on reducing the $d$-CDW excitations.

In conclusion, our results show that the $\mathrm{A}_{1 g}$ ERS peak present a constant gap to $T_{c}$ ratio $\sim 5-6$ independent of the presence of magnetic or non-magnetic impurities. Also, the $\mathrm{B}_{1 g}$ ERS peak is smeared out in the $\mathrm{Y} 123$ when the $\mathrm{Cu}^{2+}$ is substituted by small amount of non-magnetic $\mathrm{Zn}$ impurities whereas the $\mathrm{A}_{1 g}+\mathrm{B}_{2 g}$ spectra remain insensitive to the kind of impurity. These results could be qualitatively interpreted in terms of the of Zeyher and Greco's 17] theory that relates the ERS in the $\mathrm{A}_{1 g}$ and $\mathrm{B}_{1 g}$ channels to $d$-CDW and superconducting order parameters fluctuations, respectively.

\section{ACKNOWLEDGMENTS}

This work was supported by the Brazilian Agencies CNPq and FAPESP. We would like to thank A. Greco, P.G. Pagliuso and R. L. Doretto for fruitful discussions.
[1] T. Moriya and K. Ueda, Adv. Phys. 49, 555 (2000).

[2] A. Chubukov, D. Pinnes, and J. Schmalian in The Physics of Superconductors, ed. K. H. Bennermann, and J. B. Ketterson, Springer-Verlag (2003).

[3] V. Barzykin and D. Pines, Phys. Rev B 52, 13585 (1995); W. Anderson, Science 235, 1196 (1987); M. U. Ubbens and P. A. Lee, Phys. Rev. B 46, 8434 (1992); P. A. Lee and X. G. Wen, Phys. Rev. Lett. 78. 4111 (1997); J. M. Tranquada et al, Phys. Rev. Lett. 78, 338 (1997); C. Castellani, C. Di Castro, and M. Grilli, Z. Phys. B 103, 137 (1997).

[4] S. B. Dierker, M. V. Klein, G. W. Webb, and Z. Fisk, Phys. Rev. Lett. 50, 853 (1983).

[5] M. Cardona, Physica C 317, 30 (1999).

[6] I. S. Yang, M. V. Klein, S. L. Cooper, P. C. Canfield, B. K. Cho, S. I. Lee, Phys. Rev. B 62, 1291 (2000)

[7] J. W. Quilty, S. Lee, A. Yamamoto, and S. Tajima, Phys. Rev. Lett 88, 87001 (2002).

[8] A. A. Martin, V. G. Hadjiev, C. Bernhard, T. Ruf, M. Cardona, and T. Wolf, Phys. Stat. Sol. (b) 214, R21 (1999).

[9] T. P. Devereaux and D. Einzel, Phys. Rev. B 51, 16336 (1995); T. P. Devereaux et al. Phys. Rev. B 54, 12523 (1996); T. Strohm and M. Cardona, Solid State Commun. 9, 233 (1997); M. Krantz and M. Cardona, J. Low Temp. Phys. 99, 205 (1995).

[10] Y. Sidis, P. Bourges, H. F. Fong, B. Keimer, L. P. Regnault, J. Bossy, A. Ivanov, B. Hennion, P. GautierPicard, G. Collin, D. L. Millius and J. A. Aksay, Phys.
Rev. Lett 84, 5900 (2000).

[11] H. F. Fong, P. Bourges, Y. Sidis, L. P. Regnault, J. Bossy, A. Ivanov, D. L. Milius, I. A. Aksay, and B. Keimer, Phys. Rev. Lett. 82, 1939 (1999).

[12] C. T. Lin, S. X. Li, A. Mackenzie, W. Zhou, P. D. Hunneyball, W. Y. Liang, Physica C 193, 129 (1992).

[13] T. Strohm and M. Cardona, Phys. Rev B 55, 12725 (1997); B 58, 8839 (1998).

[14] J. Geerk, X. X. Xi and G. Linker, Z. Phys. B 73, 329 (1988); M. Gurvitch, J. M. valles, A. M. Cucolo, R. C. Dynes, J. P. Garno, L, F. Schneemeyer, and J. V. Wasczak, Phys. Rev. Lett. 63, 1008 (1989); A. Fournel, I. Oujia, I. Sorbier, Europhys. Lett. 6, 653 (1988).

[15] F. Venturini, U. Michelucci, T. P. Devereaux, and A. P. Kampf,Phys. Rev. B 62, 15204 (2000).

[16] Y. Gallais, A. Sacuto, P. Bourges, Y. Sidis, A. Forget, and D. Colson, Phys. Rev. Lett. 88, 177401 (2002).

[17] R. Zeyher and A. Greco, Phys. Rev. Lett. 89, 177004 (2002).

[18] E. Cappelluti and R. Zeyher, Phys. Rev. B 59, 6475 (1999).

[19] H. J. Schulz, Phys. Rev. B 39, 2940 (1989); Sudip Chakravarty, R. B. Laughlin, Dirk K. Morr, and Chetan Nayak, Phys. Rev. B 63, 94503 (2001).

[20] Raju P. Gupta and Michèle Gupta, Phys. Rev. B 59, 3381 (1999).

[21] E. Cappelluti and R. Zeyher, Europhys. Lett. 49, 487 (2000). 